D. Eisenbud, W. Vasconcelos and R. Wiegand

Nagoya Math. J.

Vol. 86 (1982), 203-209

\title{
PROJECTIVE SUMMANDS IN GENERATORS
}

\author{
DAVID EISENBUD ${ }^{1)}$, WOLMER VASCONCELOS ${ }^{1)}$ \\ AND ROGER WIEGAND ${ }^{1)}$
}

An $R$-module $M$ is a generator (of the category of modules) provided every module is a homomorphic image of a suitable direct sum of copies of $M$. Equivalently, some $M^{(k)}$ has $R$ as a summand. Except in the last section, all rings are assumed to be commutative, Noetherian domains, and modules are usually finitely generated. In this context generators are exactly those modules that have non-zero free summands locally. Of course, generators can fail to have free summands (e.g., over Dedekind domains), and we ask whether they necessarily have non-zero projective summands. The answer is "yes" for rings of dimension 1, as we point out in $\S 3$, and for the polynomial ring in one variable over a Dedekind domain. In $\S 1$ we show that for 2-dimensional rings the answer is intimately connected with the structure of projective modules. Our main result in the positive direction, Theorem 1.3, grew out of the attempt, in conversations with T. Stafford, to understand the case $R=k[x, y]$. In $\S 2$ we give examples of rings having generators with no projective summands. The last section contains miscellaneous observations, some of them on rings without chain conditions.

\section{§1. Dimension two}

The next three theorems are the heart of the paper. They will be used in the next section to answer the question raised in the introduction, for certain specific rings.

Theorem 1.1. Let $R$ be a two-dimensional regular affine domain over an algebraically closed field. These three conditions on $R$ are equivalent:

(i) Every generator has a non-zero projective summand.

Received May 16, 1980.

Revised October 8, 1980.

1) Parts of this research were supported by the National Science Foundation. 
(ii) Every projective $R$-module is the direct sum of rank 1 projectives.

(iii) Every maximal ideal of $R$ is the sum of two height-one primes.

The implication (iii) $\Rightarrow$ (ii) is proved in [MS]. (See the discussion after Corollary 6.6, and apply Theorem 1.) We will show that (i) $\Rightarrow$ (iii), and (ii) $\Rightarrow$ (i). Each of these implications holds in a more general setting, so we state them separately.

Theorem 1.2. Let $R$ be a Noetherian domain for which each generator has a non-zero projective summand. Assume either

(a) every invertible ideal is principal; or

(b) $R$ has projective cancellation.

Then every non-singular maximal ideal of height two is the sum of two invertible ideals.

Proof. Suppose $P$ is a counterexample. We claim there is a maximal ideal $Q \neq P$ of depth $\geq 2$. When $R$ is affine this is trivial; in the general case we proceed as follows: Pick $a \in P-P^{2}$, and let bars denote passage to $\bar{R}=R /(a)$. Then $\bar{R}_{\bar{P}}$ is a discrete valuation ring, and by [Ma, 15. G] $\bar{P}$ has depth 1 . Choose $b \in P$ so that $\vec{b} \notin \bar{P}^{2} \cup(\cup$ Ass $\bar{R})$. Then $(a, b)$ is an $R$-sequence and $(a, b) R_{P}=P R_{P}$. We may now choose $Q$ to be any maximal ideal in the support of $P /(a, b)$.

Note that $\operatorname{Ext}_{R}^{1}(P, Q)=\operatorname{Ext}_{R}^{2}(R / P, Q)=\operatorname{Ext}_{R_{P}}^{2}\left(R / P, R_{P}\right)=R / P$, a cyclic $R$-module. Choose an extension

$$
\text { (E) } 0 \longrightarrow Q \longrightarrow M \longrightarrow P \longrightarrow 0
$$

whose equivalence class generates $\operatorname{Ext}_{R}^{1}(P, Q)$. Clearly h. $\operatorname{dịm}_{R}(P)=1$, and $M_{P}$ is free by [S]. If $m$ is any maximal ideal different from $P$, we have $M_{\mathrm{m}} \cong Q_{\mathrm{m}} \oplus R_{\mathrm{m}}$. Thus $M$ is a generator. Note also that

$$
M \cong R \oplus Q \text { locally . }
$$

Certainly $M$ can't be projective, since otherwise $Q$ would be projective, contradicting height $(Q) \geq 2$. Since $M$ has rank 2 , we conclude that $M$ $\cong I \oplus J$, where $I$ and $J$ are ideals, with $I$ invertible. It follows from local cancellation, [E], that $J$ and $Q$ are locally isomorphic. Since depth (Q) $\geq 2$, one checks that $(J: Q)$ is invertible, and $(J: Q) Q=J$. If now (a) holds, then $J \cong Q$. From (E) we get a surjection $(u, v): R \oplus Q \longrightarrow P$, with $u \in P$ and $v Q \subseteq P$. Then $v \in(R: Q)=R$, so $v \in P$, and clearly $P=$ $u R+v R$, contradiction. 
Suppose condition (b) is satisfied. Choose a generator for the cyclic $R$-module $\operatorname{Ext}_{R}^{1}(P, R)$, say $\left(\mathrm{E}^{\prime}\right) 0 \rightarrow R \rightarrow F \rightarrow P \rightarrow 0$. It will suffice to prove that $F$ is the direct sum of two projective modules of rank 1. From [S] we know, at least, that $F$ is projective.

Form the pullback of $(\mathrm{E})$ and $\left(\mathrm{E}^{\prime}\right)$ :

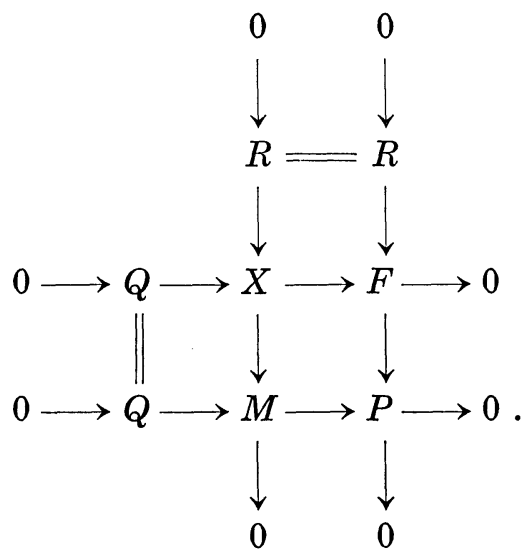

From (1) we see that $X=F \oplus Q$ is locally isomorphic to $M \oplus R$. Therefore, by [Mi], the left-hand vertical sequence splits locally, hence globally. Thus $F \oplus Q \cong I \oplus J \oplus R$, so $F \oplus Q^{* *} \cong I \oplus J^{* *} \oplus R$. But $Q^{* *}=R$, and we can cancel, to get $F \cong I \oplus J^{* *}$.

Theorem 1.3. Let $R$ be a regular Noetherian domain of dimension 2, and assume every projective $R$-module is the direct sum of rank-one projectives. Then every generator has a summand isomorphic to an invertible ideal.

Proof. Let $M$ be a generator, and choose a surjection $f: M^{(m)} \longrightarrow R$. Since the image of the transpose map $f^{t}: M \rightarrow R^{(m)}$ is still a generator, we may assume $M$ is finitely generated and torsion-free. If $M$ is an ideal of $R$, the equations that say $M$ is a generator say precisely that $M$ is invertible. Thus we assume the rank of $M$ is at least 2. Let $J=$ $\left(0: M^{* *} / M\right)$. Then $R / J$ is zero-dimensional since $M=M^{* *}$ at each prime ideal of height 1. Note $M^{* *}$ is projective, being a second syzygy of the transpose module of $M$, so write $M^{* *}=\sum_{j=1}^{n} I_{j}$, where each $I_{j}$ is an invertible ideal of $R$. We may assume, by [MS, Lemma 4.3], that $I_{j}+J=$ $R$ for all $j$. If $u \in I_{j}$, we denote by $u^{\prime}$ the element of $M^{* *}$ with $u$ in the $j^{\text {th }}$ coordinate and zeroes elsewhere. (Though technically ill-defined, the 
relevant coordinate $j$ will always be clear from the context.) Choose $e_{j} \in$ $I_{j}$, with $1-e_{j} \in J$, and note that $e_{1}^{\prime}, \cdots, e_{n}^{\prime}$ map to a basis of the free $R / J$ - module $M^{* *} / J M^{* *}$. Since $M$ is a generator, $M^{* *} / M$ is locally generated by $n-1$ elements, hence globally $(n-1)$-generated. We can choose $a_{j}$ $\in I_{n}$ so that the images of $e_{j}^{\prime}+a_{j} e_{n}^{\prime}, 1 \leq j \leq n-1$, generate $M^{* *} / M$. Choose $b_{j} \in I_{j}$ so that $x=e_{n}^{\prime}+\sum_{j} b_{j}\left(e_{j}^{\prime}+a_{j} e_{n}^{\prime}\right)$ is in $M$. Consider the matrix

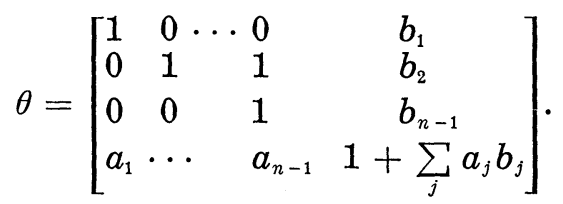

This has its $i k$ entry in $I_{i} I_{k}^{-1}$, and so $\theta\left(M^{* *}\right) \subseteq M^{* *}$. Since the determinant is $1, \theta$ is an automorphism locally, and thus is an automorphism. The proof will be complete if we can show that $\theta$ carries the direct factor $I_{n}$ into $M$.

First we note that $\theta\left(e_{n}^{\prime}\right) \in M$. To see this, write

$$
\begin{aligned}
\theta\left(e_{n}^{\prime}\right) & =e_{n} \sum_{j} b_{j}^{\prime}+\left(1+\sum_{j} a_{j} b_{j}\right) e_{n}^{\prime} \\
& =\sum_{j}\left(e_{n}-e_{j}\right) b_{j}^{\prime}+\sum_{j} b_{j} e_{j}^{\prime}+\left(1+\sum_{j} a_{j} b_{j}\right) e_{n}^{\prime} \\
& =\sum_{j}\left(e_{n}-e_{j}\right) b_{j}^{\prime}+x .
\end{aligned}
$$

But $x \in M$, and $e_{n}-e_{j}=\left(1-e_{j}\right)-\left(1-e_{n}\right) \in J=\left(M: M^{* *}\right)$. Finally, for $y \in I_{n}$, we have $\theta\left(y^{\prime}\right)=y \theta\left(e_{n}^{\prime}\right)+\left(1-e_{n}\right) \theta\left(y^{\prime}\right)$, which is in $M$, again because $1-e_{n} \in J$.

The only part of Theorem 1.1 that remains to be proved is the implication (i) $\Rightarrow$ (iii). But this follows from Theorem 1.2 and the projective cancellation theorem of [MS].

\section{§ 2. Examples}

We apply the results of $\S 1$ to some specific classes of rings.

Example 2.1. Let $D$ be a Dedekind domain and set $R=D[X]$. By (1.3) every generator over $R$ has a projective summand of rank 1 .

ExAmple 2.2. Let $R$ be the coordinate ring of the real 2-sphere. The real maximal ideals of $R$ require 3 generators, [GW], and $R$ is a unique factorization domain. Therefore, by (1.2), $R$ has a generator with no projective summands. 
For the rest of this section, we fix the following notation: $X$ is a smooth projective surface over $C$, and $V=\operatorname{spec}(R)$ is an arbitrary affine open subset of $X$. Let $q=p_{g}-p_{a}$ be the irregularity of $X$. If $X$ is a complete intersection (in particular, if $X \subset P^{3}$ ), then $q=0$.

Theorem 2.3. Assume either (a) $X$ is a rational surface; or

(b) $X$ is the product of two curves; or

(c) $p_{g}=0$ and $q>0$.

Then every generator over $R$ has a non-zero projective summand.

Proof. In cases (a) and (c), every projective $R$-module is the direct sum of a free module and an ideal, by [MS, Theorem 2 and Corollary 7.5]. In case (b), condition (iii) of Theorem 1.1 is satisfied.

THEOREM 2.4. If $q=0$ and $p_{g}>0$, then $R$ has a generator with nonzero projective summands.

Proof. By [MS, Theorem 6.1], $R$ has an indecomposable projective module of rank 2 .

Example 2.5. Suppose $X$ is a smooth surface in $P^{3}$ defined by an irreducible homogeneous polynomial of degree $d$. If $d \leq 3, X$ is a rational surface, while if $d \geq 4$ we have $p_{a}=p_{g}>0$. Thus generators over $R$ have projective summands if and only if $d \leq 3$.

\section{§3. Miscellany}

In this section we relax the chain conditions imposed in the introduction.

Proposition 3.1. Let $R$ be a commutative integral domain of dimension 1. Then every finitely presented generator has a rank 1 projective summand.

Proof. Let $M$ be a finitely presented generator. As in the proof of (1.3) we may assume the torsion-free rank of $M$ is at least 2. Since $M$ is finitely presented there is a map $\phi=(f, g): M \rightarrow R^{(2)}$ such that $\phi \otimes 1_{K}$ is onto. Let $I=(0:$ Coker $\phi)$. Now $R / I$ is zero-dimensional, and we can patch together maps $f_{i}: M \rightarrow R$ to get a single map $h: M \rightarrow R$ that is onto modulo $I$. We wish to replace $g$ by a map $g^{\prime}$ such that $\phi^{\prime}=\left(f, g^{\prime}\right)$ still maps $M \otimes K$ onto $K^{(2)}$ and for every maximal ideal $p$ either $f_{p}$ or $g_{p}^{\prime}$ is onto. If the image of $(f, h) \otimes 1_{K}$ is two-dimensional take $g^{\prime}=h$. Other- 
wise, choose $0 \neq r \in I$, and let $g^{\prime}=h+r g$. Then $C=$ Coker $\phi^{\prime}$ is torsion, and since $\operatorname{Im} \phi^{\prime}$ splits off a free summand of $R^{(2)}$ at each maximal ideal, $C$ is locally cyclic. But then $C$ is cyclic, being a module over a zerodimensional ring. Let $u, v$ be a basis for $R^{(2)}$. Since zero-dimensional rings have 1 in the stable range, there is an element $a \in R$ such that the image of $u+a v$ generates $C$. Then $v-b(u+a v) \in \operatorname{Im} \phi^{\prime}$ for some $b \in R$. We now have a basic element of $R^{(2)}$ in $\operatorname{Im} \phi^{\prime}$, so $M$ maps onto $R$.

We note that if $M$ is a generator over a commutative ring $R$ of dimension $d$, then $M^{(d+1)}$ has a summand isomorphic to $R$. When $R$ has Noetherian spectrum this follows from a standard general position argument. In the general case, we appeal to the following:

Proposition 3.2. Let $R$ be a commutative ring of dimension $d$, and let $M$ and $N$ be $R$-modules, with $N$ finitely generated. Suppose, for each maximal ideal $p$ of $R$, there is a map $f: M \rightarrow N$ such that $f_{p}$ is onto. Then there is a surjection $M^{(d+1)} \rightarrow N$.

We omit the proof, as it is a minor modification of the proof of [VW, (2.1)].

Finally, we suggest a method of generalizing Theorem 1.3 to certain singular rings.

Proposition 3.3. Let $A \subseteq B$ be integral domains with the same quotient field, and let $I$ be an ideal of both $A$ and $B$ such that Pic $(B / I)$ $=0$. If $A / I$ and $B$ both have the property that each finitely generated generator has a rank 1 projective summand, then the same is true over $A$.

Proof. Let $M$ be a finitely generated generator over $A$. We may assume $M$ is torsionfree. The diagram

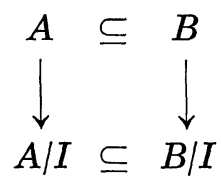

is simultaneously a pullback and a pushout. When we tensor everything with $M$ the top arrow is still a monomorphism, so the resulting pushout is also pullback. Choose rank 1 projective modules $P$ and $Q$ over $A / I$ and $B$, respectively, and surjections $M / I M \longrightarrow P, M \otimes B \longrightarrow Q$. Since Pic $(B / I)=0, P \otimes B / I \cong Q \otimes B / I$, and any choice of an isomorphism makes the pullback of $P$ and $Q$ a rank 1 projective summand of $M$. 
For example, take $A=k\left[X^{2}, X Y, Y\right], B=k[X, Y]$ and $I=Y k[X, Y]$. Using (3.1), (1.3) and (3.3) we see that every generator over $A$ has a rank 1 projective summand.

\section{REFERENCES}

[E] E. G. Evans, Jr., Krull-Schmidt and cancellation over local rings, Pacific J. Math., 46 (1973), 115-121.

[GW] A. Geramita and C. Weibel, Ideals with trivial conormal bundle, Canadian J. Math., 32 (1980), 210-218.

[Ma] H. Matsumura, Commutative Algebra, Benjamin, New York 1970.

[Mi] T. Miyata, Note on direct summands of modules, J. Math. Kyoto Univ., 7 (1967), 65-69.

[MS] M. P. Murthy and R. G. Swan, Vector bundles over affine surfaces, Invent. Math., 36 (1976), 125-165.

[S] J. P. Serre, Sur les modules projectifs, Sem. Dubreil-Pisot, 14, No. 2 (1960-1961).

[VW] W. Vasconcelos and R. Wiegand, Bounding the number of generators of a module, Math. Z., 164 (1978), 1-7.

Brandeis University

Waltham, MA 02154

Rutgers University

New Brunswick, $N J$

08903

University of Nebraska

Lincoln, NE 68588 